\title{
Modeling the gravitational wave signature of neutron star black hole coalescences
}

\author{
Jonathan E. Thompson $\odot,{ }^{1}$ Edward Fauchon-Jones $\odot,{ }^{1}$ Sebastian Khan $\odot,{ }^{1,2,3}$ Elisa Nitoglia $\odot,{ }^{4,5}$ \\ Francesco Pannarale $\odot^{4,5}$ Tim Dietrich $\odot,{ }^{6,7}$ and Mark Hannam ${ }^{1,4}$ \\ ${ }^{1}$ School of Physics and Astronomy, Cardiff University, Queens Buildings, Cardiff, \\ CF24 3AA, United Kingdom \\ ${ }^{2}$ Max Planck Institute for Gravitational Physics (Albert Einstein Institute), \\ Callinstr. 38, 30167 Hannover, Germany \\ ${ }^{3}$ Leibniz, Universität Hannover, D-30167 Hannover, Germany \\ ${ }^{4}$ Dipartimento di Fisica, Università di Roma "Sapienza", Piazzale A. Moro 5, I-00185, Roma, Italy \\ ${ }^{5}$ INFN Sezione di Roma, Piazzale A. Moro 5, I-00185, Roma, Italy \\ ${ }^{6}$ Nikhef, Science Park, 1098 XG Amsterdam, Netherlands \\ ${ }^{7}$ Institut für Physik und Astronomie, Universität Potsdam, Haus 28, Karl-Liebknecht-Str. 24/25, \\ 14476, Potsdam, Germany
}

(Received 2 March 2020; accepted 19 May 2020; published 26 June 2020)

\begin{abstract}
Accurate gravitational-wave (GW) signal models exist for black hole binary $(\mathrm{BBH})$ and neutron-star binary (BNS) systems, which are consistent with all of the published GW observations to date. Detections of a third class of compact-binary systems, neutron-star black hole (NSBH) binaries, have not yet been confirmed, but are eagerly awaited in the near future. For NSBH systems, GW models do not exist across the viable parameter space of signals. In this work we present the frequency-domain phenomenological model, PhenomNSBH, for GWs produced by NSBH systems with mass ratios from equal-mass up to 15, spin on the black hole $(\mathrm{BH})$ up to a dimensionless spin of $|\chi|=0.5$, and tidal deformabilities ranging from 0 (the $\mathrm{BBH}$ limit) to 5000. We extend previous work on a phenomenological amplitude model for NSBH systems to produce an amplitude model that is parametrized by a single tidal deformability parameter. This amplitude model is combined with an analytic phase model describing tidal corrections. The resulting approximant is compared to publicly available NSBH numerical-relativity simulations and hybrid waveforms constructed from numerical-relativity simulations and tidal inspiral approximants. For most signals observed by second-generation ground-based detectors, it will be difficult to use the GW signal alone to distinguish single NSBH systems from either BNSs or BBHs, and therefore to unambiguously identify an NSBH system.
\end{abstract}

DOI: 10.1103/PhysRevD.101.124059

\section{INTRODUCTION}

Stellar-mass compact-binary coalescences have been the source of all current gravitational-wave (GW) observations made by the Advanced LIGO [1] and Advanced Virgo detectors [2]. The data collected during the first and second observing runs are publicly available $[3,4]$, and analyses of it have been published in several GW catalogs [5-8]. The compact-binary mergers expected to be observed by current ground-based detectors come in three varieties: black hole binaries (BBHs), neutron-star binaries (BNSs), and binaries that consist of one black hole and one neutron star (NSBHs). The majority of GW signals detected so far comes from BBH mergers, with two detections, GW170817 [9] and GW190425 [10], inferred to be from BNS mergers. Although the GW signals from these two events are also consistent with NSBH mergers, e.g., [11,12], this class of merger has yet to be unambiguously observed.
To extract physical information from GW signals, template waveforms constructed from theoretical models are compared with the data using a Bayesian framework. Much of the previous waveform modeling efforts have focused successfully on BBHs-for examples of recent BBH waveform models, see SEOBNRv4HM [13], PhenomPv3HM [14,15], and surrogates NRSur7dq4 [16] and NRHybSur3DQ8 [17]. These BBH waveform models do not capture the changes to the waveform morphology introduced when one or both of the binary companions is a neutron star (NS). One effect is a shift to the waveform phase that arises from tidal deformation of the NS during the inspiral of the two bodies [18]. This shift has been the focus of recent research into BNS waveform modeling efforts, and has produced several available models: TEOBResumS [19], SEOBNRv4T [20-22], and the NRTidal models [23-25]. These phase corrections have been sufficient in observations to date, because disruption of the NSs 
produces changes in the $\mathrm{GW}$ amplitude at high frequency [26-28], where the detectors have been largely insensitive to the merger and postmerger BNS signal $[29,30]$.

In signals from NSBH systems, the phase shift during the inspiral stage due to NS tidal deformation is present, but it is unlikely that it will be observable with current detectors [31]. Further, and in contrast to BNS signals, merger and postmerger dynamics in NSBH systems are potentially accessible to current ground-based detectors due to these systems' potential for higher total masses, which can shift the GW signal at merger to a more sensitive part of the frequency band. As the mass-ratio of the system increases, the merger morphology of the waveform can range from total disruption of the NS, in which case the amplitude of the waveform is exponentially suppressed at high frequency [32], to nondisruptive signals for which the waveform is comparable to a $\mathrm{BBH}$ waveform, where the high-frequency amplitude is governed by the ringdown of the companion black hole (BH) [33]. Observations of the merger signal in an NSBH could allow us to place tighter constraints on the NS equation of state (EOS) [34-36] and identify its source as an NSBH binary. Of the waveform models existing currently, LEA [37] and the upgraded LEA+ models are the only existing NSBH waveform models that include an NSBH-specific merger morphology and are calibrated against NSBH NR waveforms. While effective in their shared calibration range, their parameter space coverage is limited, in particular only to mass ratios between 2 and 5 .

The aim of this work is to produce a new NSBH model called PhenomNSBH that combines an approximate reparametrization of the NSBH amplitude model described by [38] with the state-of-the-art tidal phase model described in [25]. As with previous work, the new model supports a spinning $\mathrm{BH}$ with spin vector parallel to the orbital angular momentum of the system and a nonspinning NS. Furthermore, we simplify the previous amplitude modeling efforts by replacing dependence on the NS EOS with a single tidal deformability parameter. This change is essential to allow our new model to be used for parameter estimation. With these changes to the amplitude model and the integration of an improved phase description, our new model is valid over a larger parameter space, and it is capable of generating accurate waveforms from equal mass up to mass-ratio 15. At high mass ratios, the NS merges with the $\mathrm{BH}$ before disrupting, and the $\mathrm{GW}$ signal approaches that of an equivalent BBH. As we show in Sec. III, beyond mass-ratio 8 a $\mathrm{BBH}$ model will be sufficient for observations with a signal-to-noise ratio less than 300 .

In Sec. II we describe and outline the waveform model PhenomNSBH presented in this paper, which is implemented as IMRPhenomNSBH in the open-source software package LALSuite [39]. To assess the PhenomNSBH model, we compare it against numerical-relativity (NR) data for various
NSBH systems in Sec. III, presenting alongside the same comparisons for other relevant waveform models, and we identify the regions of parameter space where an NSBH model will be necessary to prevent measurement biases. Finally we conclude with Sec. IV, where we summarize our results and discuss directions for future work. In the remaining sections of this paper geometric units are used such that $G=c=1$.

\section{MODELING NEUTRON STAR-BLACK HOLE WAVEFORMS}

In this section we present a model for the GW signal emitted by an NSBH binary system that consists of a nonspinning NS and a $\mathrm{BH}$ with spin angular momentum $\mathbf{S}_{\mathrm{BH}}$ parallel to the orbital angular momentum $\mathbf{L}$ of the system. Such a system may be parametrized by four intrinsic parameters: $M$, the total mass of the system, $M=M_{\mathrm{BH}}+M_{\mathrm{NS}}$, where $M_{\mathrm{BH}}$ and $M_{\mathrm{NS}}$ are the component masses of the BH and NS, respectively; $q$, the mass ratio of the system where $q=M_{\mathrm{BH}} / M_{\mathrm{NS}} \geq 1 ; \chi$, the dimensionless spin of the BH given by $\chi=\mathbf{S}_{\mathrm{BH}} \cdot \hat{\mathbf{L}} / M_{\mathrm{BH}}^{2}$; and $\Lambda$, the dimensionless NS tidal deformability parameter $[18,40]$ defined in terms of the quadrupolar Love number, $k_{2}$, and compactness $C=M_{\mathrm{NS}} / R_{\mathrm{NS}}$ of the NS,

$$
\Lambda=\frac{2}{3} \frac{k_{2}}{C^{5}} .
$$

We encapsulate these four parameters in the vector $\boldsymbol{\theta}=(M, q, \chi, \Lambda)$. Note that, unlike BBH models, the total mass $M$ cannot be separated as a scaling factor due to the scale-dependent effects that arise in the waveform from the presence of the NS.

We seek a model of the complex strain in the frequency domain, $\tilde{h}(f ; \boldsymbol{\theta}, \vartheta, \varphi)$, where the extrinsic parameters $(\vartheta, \varphi)$ represent the orientation of the system with respect to a distant observer. The strain may be written as an expansion in spin-weighted spherical harmonics ${ }_{-2} Y_{\ell m}(\vartheta, \varphi)$. For the first step in this preliminary model, we follow previous phenomenological models $[38,41-43]$ and focus only on the dominant $(\ell,|m|)=(2,2)$ multipole moments, i.e.,

$$
\begin{aligned}
\tilde{h}(f ; \boldsymbol{\theta}, \vartheta, \varphi) & =\sum_{\ell, m} \tilde{h}_{\ell m}(f ; \boldsymbol{\theta})_{-2} Y_{\ell m}(\vartheta, \varphi) \\
& \approx \sum_{m= \pm 2} \tilde{h}_{2 m}(f ; \boldsymbol{\theta})_{-2} Y_{2 m}(\vartheta, \varphi) .
\end{aligned}
$$

The $\tilde{h}_{22}$ multipole moment is further decomposed in terms of an amplitude $A$ and phase $\phi$,

$$
\tilde{h}_{22}(f ; \boldsymbol{\theta})=A(f ; \boldsymbol{\theta}) e^{-i \phi(f ; \boldsymbol{\theta})},
$$

and we relate $\tilde{h}_{2-2}(f)=\tilde{h}_{22}^{*}(-f)$, where $*$ denotes complex conjugation. Higher multipoles are also necessary 
for unbiased parameter measurements for systems with $q \geq 3[44,45]$. A quadrupole-only model is however sufficient to capture the broad phenomenology of the signal from an NSBH system including the effects of tidal disruption, and for all of the conclusions that we draw in this work. Using the calibrated higher-mode BBH model IMRPhenomXHM [46], we estimate that the first subdominant multipole moment contributes only an estimated $10 \%-15 \%$ of additional signal power when measured over the disruptive and mildly disruptive region of the NSBH parameter space, due to the relatively low total mass of the NSBH system. We will discuss further extensions in Sec. IV.

In the text that follows, we outline in detail how the amplitude and phase are modeled for an NSBH system.

\section{A. Amplitude model}

To create an amplitude model for PhenomNSBH we start from the NSBH amplitude description of Pannarale et al. in [38]. This model describes an amplitude based on the aligned-spin BBH waveform amplitude of PhenomC [41], which depends on three intrinsic parameters $(M, q, \chi)$ and an explicit choice of a NS equation of state (EOS). Four choices of EOS were used in its calibration, listed in order of increasing softness, i.e., decreasing tidal deformability: $2 \mathrm{H}, \mathrm{H}, \mathrm{HB}$, and B [47]. Given an EOS and NS gravitational mass $M_{\mathrm{NS}}$ (assuming $M_{\mathrm{NS}} \leq M_{\mathrm{BH}}$ ), the amplitude model of Pannarale et al. integrates the Tolman-Oppenheimer-Volkoff equations [48-50] to find the NS radius $R_{\mathrm{NS}}$ and baryonic mass $M_{b, \mathrm{NS}}$ associated with its gravitational mass. From the mass and radius, the NS compactness is computed via $C=M_{\mathrm{NS}} / R_{\mathrm{NS}}$.

While determination of the NS EOS may be possible after several detections [51], it is more practical for our waveform model to not be directly dependent on the EOS. To this end, we replace the dependency of the amplitude model on the EOS with a dependency on the dimensionless tidal deformability $\Lambda$, outlined in the Appendix B. With these augmentations made to the original amplitude model, we have a working amplitude for an aligned-spin NSBH system with dependence on the four intrinsic parameters $(M, q, \chi, \Lambda)$. Based on the workflows provided in Refs. $[38,52]$, the amplitude model is evaluated using the following steps:

(1) Calculate the NS compactness $C$

Evaluate Eq. (B1) to calculate compactness $C(\Lambda)$ of the NS.

(2) Calculate the tidal disruption frequency $f_{\text {tide }}$

Evaluate Eq. (A1) to calculate the tidal disruption frequency $f_{\text {tide }}(q, \chi, C)$.

(3) Calculate the baryonic mass ratio $M_{b, \text { torus }} / M_{b, \mathrm{NS}}$

Evaluate Eq. (A4) to calculate the baryonic mass ratio of the NS. This model depends only on the torus remnant baryonic mass $M_{b \text {,torus }}$ and the baryonic mass $M_{b, \mathrm{NS}}$ of the isolated NS at rest through expressions of the form $M_{b, \text { torus }} / M_{b, \mathrm{NS}}$. As such it is not necessary to calculate an explicit value for $M_{b, \mathrm{NS}}$, which was required by [38].
(4) Calculate remnant BH properties $\left(\chi_{f}, M_{f}\right)$

Evaluate Eq. (A6) to calculate the final spin $\chi_{f}(\eta, \chi, \Lambda)$ and final mass $M_{f}(\eta, \chi, \Lambda)$ of the remnant black hole, where $\eta=q /(1+q)^{2}$ is the symmetric mass ratio.

(5) Calculate the remnant $\mathbf{B H}$ quantities $\left(f_{\mathrm{RD}}, Q\right)$

Evaluate Eqs. (A9) and (A10) to calculate the ringdown frequency $f_{\mathrm{RD}}\left(M_{f}, \chi_{f}\right)$ and quality factor $Q\left(\chi_{f}\right)$.

(6) Calculate merger-type dependent quantities

Calculate the merger-type dependent quantities $\left(\epsilon_{\text {tide }}, \epsilon_{\text {ins }}, \sigma_{\text {tide }}, \tilde{f}_{0}, \tilde{f}_{1}, \tilde{f}_{2}\right)$ using the conditions on $f_{\text {tide }}, f_{\mathrm{RD}}$, and the magnitude of $M_{b \text {,torus }}$, and expressions provided in Table I.

(7) Calculate non-merger-type dependent quantities

Evaluate Eq. (A24) to calculate the phenomenological parameters $\gamma_{1}, \delta_{1}$, and $\delta_{2}$. Evaluate Eqs. (A19) and (A22) to calculate the phenomenological correction parameters $\gamma_{1}^{\prime}$ and $\delta_{2}^{\prime}$, respectively. While $\delta_{1}$ and $\delta_{2}^{\prime}$ are not explicitly dependent on any mergertype dependent quantities, they are not required if the onset of tidal disruption happens before the ringdown frequency is reached.

(8) Evaluate the amplitude

Evaluate the amplitude $A(f ; \boldsymbol{\theta})$,

$$
\begin{aligned}
A(f)= & A_{\mathrm{PN}}(f) \omega_{\tilde{f}_{0}, 0.015+\sigma_{\text {tide }}}(f) \\
& +\gamma_{1}^{\prime} f^{5 / 6} \omega_{\tilde{f}_{1}, 0.015+\sigma_{\text {tide }}}^{-}(f) \\
& +A_{\mathrm{RD}}(f) \omega_{\tilde{f}_{2}, 0.015+\sigma_{\text {tide }}}^{+}(f),
\end{aligned}
$$

where $\omega_{f_{0}, d}^{ \pm}$is defined by Eq. (A23) and $A_{\mathrm{PN}}$ and $A_{\mathrm{RD}}$ are defined by Eqs. (A18) and (A20), respectively. We have suppressed all explicit parametrization in the component functions of the amplitude $A$ for legibility.

The specific parameters used in the amplitude model change based on the classification of the system, as detailed in Table I. As described in Refs. [38,52], the type of merger modeled by the amplitude varies between nondisruptive and disruptive cases, with the conditions identifying each case listed at the top of Table I. We now briefly summarize the various cases allowed for in the model. When the computed tidal disruption frequency, $f_{\text {tide }}$, exceeds the ringdown frequency, $f_{\mathrm{RD}}$, and the model predicts no remnant torus mass, $M_{b \text {,torus }}=0$, the signal is classified to be nondisruptive, as it is assumed that the binary merges before the NS is tidally disturbed to the point of disruption. If $f_{\text {tide }}<f_{\mathrm{RD}}$, the effects of disruption appear earlier in the waveform and the system transitions toward disruption. In this case, the torus mass remnant is used to distinguish between systems that disrupt early and form a remnant mass disk, called disruptive systems, and those that disrupt late in the inspiral and no disk forms, labeled mildly disruptive. The fourth case in the table, when $f_{\text {tide }} \geq f_{\mathrm{RD}}$ and the system predicts a nonzero torus 
TABLE I. Summary of merger type dependent components of the amplitude model. For the definitions of $x_{\mathrm{ND}}, x_{\mathrm{ND}}^{\prime}, x_{\mathrm{D}}$ and $x_{\mathrm{D}}^{\prime}$, see Eqs. (A14)-(A17) in Appendix A. Note that all applications of window functions $\omega^{ \pm}$for merger-type dependent quantities are a factor of 2 smaller to correct for a typographical error in [38]. The adjusted ringdown frequency is defined as $\tilde{f}_{\mathrm{RD}}=0.99 \times 0.98 f_{\mathrm{RD}}$ for $\Lambda>1$ and $\tilde{f}_{\mathrm{RD}}=0.98 f_{\mathrm{RD}}$ for $\Lambda=0$ with a smooth interpolation given by Eq. (A13).

\begin{tabular}{|c|c|c|c|c|}
\hline \multirow[b]{3}{*}{ Merger type } & $\begin{array}{c}\text { Nondisruptive } \\
\text { (no torus remnant) }\end{array}$ & $\begin{array}{l}\text { Mildly disruptive } \\
\text { (torus remnant) }\end{array}$ & $\begin{array}{l}\text { Mildly disruptive } \\
\text { (no torus remnant) }\end{array}$ & $\begin{array}{c}\text { Disruptive } \\
\text { (torus remnant) }\end{array}$ \\
\hline & \multicolumn{2}{|c|}{$f_{\text {tide }} \geq f_{\mathrm{RD}}$} & \multicolumn{2}{|l|}{$f_{\text {tide }}<f_{\mathrm{RD}}$} \\
\hline & $M_{b, \text { torus }}=0$ & $M_{b, \text { torus }}>0$ & $M_{b, \text { torus }}=0$ & $M_{b, \text { torus }}>0$ \\
\hline$\epsilon_{\text {tide }}$ & \multicolumn{2}{|c|}{$\omega_{x_{1}, d_{1}}^{+}\left(x_{\mathrm{ND}}\right)\left[x_{1}=-0.0796251, d_{1}=0.0801192\right]$} & 0.0 & 0.0 \\
\hline$\epsilon_{\mathrm{ins}}$ & 1.0 & $1.29971-1.61724 x_{D}$ & $1.29971-1.61724 x_{D}$ & $1.29971-1.61724 x_{D}$ \\
\hline$\sigma_{\text {tide }}$ & \multicolumn{2}{|c|}{$\omega_{x_{2}, d_{2}}^{-}\left(x_{\mathrm{ND}}^{\prime}\right)\left[x_{2}=-0.206465, d_{2}=0.226844\right]$} & $\left(\omega_{x_{2}, d_{2}}^{-}\left(x_{\mathrm{ND}}^{\prime}\right)+0.137722-0.293237 x_{\mathrm{D}}^{\prime}\right) / 2$ & $0.137722-0.293237 x_{\mathrm{D}}^{\prime}$ \\
\hline$\tilde{f}_{0}$ & $\tilde{f}_{\mathrm{RD}}$ & $\epsilon_{\mathrm{ins}} \tilde{f}_{\mathrm{RD}}$ & {$\left[(q-1) \tilde{f}_{\mathrm{RD}}+\epsilon_{\mathrm{ins}} f_{\mathrm{tide}}\right] / q$} & $\epsilon_{\text {ins }} f_{\text {tide }}$ \\
\hline$\tilde{f}_{1}$ & $\tilde{f}_{\mathrm{RD}}$ & $\epsilon_{\mathrm{ins}} \tilde{f}_{\mathrm{RD}}$ & {$\left[(q-1) \tilde{f}_{\mathrm{RD}}+f_{\text {tide }}\right] / q$} & $f_{\text {tide }}$ \\
\hline$\tilde{f}_{2}$ & $\tilde{f}_{\mathrm{RD}}$ & $\tilde{f}_{\mathrm{RD}}$ & $\cdots$ & $\cdots$ \\
\hline
\end{tabular}

mass remnant, is a result of shortcomings in the fitting formulas [38], and has not arisen in our dense sampling of waveforms across the broad parameter space listed in this paper.

The torus mass and ringdown conditions that partition the parameter space into the different merger types are nonlinear in the intrinsic parameters of PhenomNSBH, and the boundaries between the different regions of the model cannot be explicitly written. Figure 2 provides an example of the transition between these cases as the mass-ratio of the system increases for a fixed tidal deformability and NS mass. For a full discussion on the distribution of merger types across the parameters please see [38]. A more detailed description of the amplitude model workflow is given in Appendix A. For full details of the amplitude model, along with the different merger types, we direct the reader to Sec. IV of Ref. [38].

\section{B. Phase model}

In addition to a proper amplitude description, we need to model the GW phase $\phi$ for the NSBH coalescence in such a way that it provides an accurate description within a large region of the parameter space and incorporates tidal effects imprinted in the signal. As a $\mathrm{BBH}$ baseline, we use the frequency-domain phase approximant from PhenomD $[42,43]$. This model allows for a description of $\mathrm{BBH}$ systems up to mass ratios of $q \leq 18$ and alignedspin components up to $|\chi| \leq 0.8$. We augment this $\mathrm{BH}$ baseline with tidal effects modeled within the NRTidal approach [23,24], using the newest version as described in Ref. [25]. The NRTidal phase model includes matter effects in the form of a closed-form, analytical expression, combining post-Newtonian knowledge with EOB and NR information. While this model was designed to be an accurate phase model for BNS systems, recent work [29] has shown that it is also a valid description in the NSBH limit.

\section{ANALYSIS OF MODEL}

To quantify the effectiveness of our model at reproducing NSBH waveforms, we compare against a selection of NR NSBH waveforms produced by the SXS Collaboration $[29,33,53]$ with simulation parameters listed in Table II. To carry out these comparisons, it is useful to

TABLE II. SXS waveforms [29,33,53] and their parameters used for comparisons and in making the hybrids. Along with the name given in the SXS public catalog, we also list an abbreviated name given to each waveform in this paper.

\begin{tabular}{llllllll}
\hline \hline Name & \multicolumn{1}{c}{ SXS name } & $q$ & $M_{\mathrm{BH}}$ & $M_{\mathrm{NS}}$ & $\chi_{\mathrm{NS}}$ & $\Lambda$ & \multicolumn{1}{c}{ Merger type } \\
\hline q1a0 & SXS:BHNS:0004 & 1 & 1.4 & 1.4 & 0 & 791 & Disruptive \\
q1.5a0 & SXS:BHNS:0006 & 1.5 & 2.1 & 1.4 & 0 & 791 & Disruptive \\
q2a0 & SXS:BHNS:0002 & 2 & 2.8 & 1.4 & 0 & 791 & Disruptive \\
q3a0 & SXS:BHNS:0003 & 3 & 4.05 & 1.35 & 0 & 607 & Mildly disruptive \\
q6a0 & SXS:BHNS:0001 & 6 & 8.4 & 1.4 & 0 & 525 & Nondisruptive \\
q1a2 & SXS:BHNS:0005 & 1 & 1.4 & 1.4 & -0.2 & 791 & Disruptive \\
q2a2 & SXS:BHNS:0007 & 2 & 2.8 & 1.4 & -0.2 & 791 & Disruptive \\
\hline \hline
\end{tabular}


introduce the notion of the overlap between two waveforms $h_{1}$ and $h_{2}$,

$$
\left\langle h_{1} \mid h_{2}\right\rangle=4 \Re \int_{f_{1}}^{f_{2}} \frac{\tilde{h}_{1}(f) \tilde{h}_{2}^{*}(f)}{S_{n}(f)} \mathrm{d} f,
$$

which is the functional inner-product weighted by the detector noise power-spectral density, $S_{n}(f)$, taken for this work to be the Advanced LIGO zero-detuned, high-power (AZDHP) noise curve [54], which is the current goal for the detector's design sensitivity. By maximizing the normalized overlap over phase $\left(\phi_{c}\right)$ and time $\left(t_{c}\right)$ shifts to $h_{1}$, one determines the faithfulness with which $h_{1}$ represents $h_{2}$,

$$
\mathcal{F}=\max _{\phi_{c}, t_{c}} \frac{\left\langle h_{1}\left(\phi_{c}, t_{c}\right) \mid h_{2}\right\rangle}{\left\|h_{1}|||| h_{2}\right\|},
$$

where $\|h\|^{2}=\langle h \mid h\rangle$.

In the following subsection we compare PhenomNSBH with publicly available numerical relativity waveforms for systems with nonspinning black holes. As there are no publicly available NR waveforms with nonzero black hole spin, such as were included in the set of simulations used in the calibration of both the LEA+ model [37] and the amplitude model [38], we first perform a comparison with LEA+ to analyze the faithfulness of the model including when the black hole has spin. We note that there is a small region above $q=5$ for high BH spin and high tidal deformability where disruption may occur. This represents a region of extrapolation for the amplitude fits; while we believe that this extrapolation is well-behaved and reasonable, there are currently no NR simulations against which we can test the model in this small region of parameter space.

The original LEA model was constructed as a phenomenological NSBH model from baseline PhenomC [41] and SEOBNR [55] BBH waveform models. Additions to the BBH models were made to include tidal post-Newtonian (PN) terms during the inspiral, and a taper was applied to the merger contributions of the waveform that was calibrated against NSBH NR waveforms. The LEA+ model was introduced as an improvement to the LEA model by substituting a reduced-order model of SEOBNRv2 [56] for the underlying BBH waveform. The LEA+ model is calibrated for NS masses ranging between 1.2-1.4 $M_{\odot}$, massratios $q \in[2,5]$, and $\mathrm{BH}$ spins $-0.5 \leq \chi \leq 0.75$. To perform the comparison, we generate waveforms across the overlapping parameter spaces covered by the calibration ranges of LEA+ and PhenomNSBH and compute the faithfulness between waveforms generated using identical parameters. The results show good agreement between the models, with $\mathcal{F}>0.99$. The comparison only deviates noticeably when $\chi<-0.4$, where the faithfulness drops to 0.98 .

Finally, we remark here that the testing done against numerical relativity is performed with simulations utilizing tidal deformabilities below 1000. While the amplitude and phase models were individually calibrated with simulations where $\Lambda$ extends to above 4000, which motivates the coverage of $\Lambda$ that we provide for this model, this calibration of the amplitude was limited in mass-ratio to between 2-5 and for NS with a limited range of masses. The good agreement with simulation $\mathrm{q} 1 \mathrm{a} 0$ included in the NR comparisons below with a tidal deformability of 791 demonstrates the ability for the model to extrapolate to equal-mass configurations; however we may expect physical effects to dominate at e.g., high tidal deformability and either equal mass or high NS mass, which have not been captured by this calibration, and we would recommend caution when using the model in this regime.

\section{A. Comparison to numerical relativity}

NR simulations typically cover the last orbits before coalescence. For the NSBH NR waveforms we consider in validating the model, the typical starting GW frequency is between $300-400 \mathrm{~Hz}$ and covers between 10 and 16 orbits before merger. Currently Advanced LIGO and Virgo are sensitive to signals starting around $20 \mathrm{~Hz}$, which for a true signal will include on the order of $10^{3}$ orbits prior to merger, and therefore the NR waveforms used here are missing a large portion of the inspiral signal [57]. We will address this issue by constructing hybrid waveforms for comparison against the model; the results of a comparison against hybrid waveforms can be found in Sec. III B. We first compare against the NR data directly in order to assess

TABLE III. The computed faithfulness between the seven SXS NSBH numerical relativity waveforms and the waveform approximants PhenomnsBH, PhenomD, PhenomDnRT, SEOBnRv4T, SEOBnRv4NRT, and LEA+. We compute two sets of matches. The first uses the Advanced LIGO zero-detuning, high-power noise curve and second, in parentheses, uses a flat noise curve. The frequency range used to compute the matches cover the entire bandwidth of the NR data.

\begin{tabular}{lcccccc}
\hline \hline Sim Name & PhenomNSBH & PhenomD & PhenomDNRT & SEOBNRv4NRT & SEOBNRv4T & LEA+ \\
\hline q1a0 & $0.988(0.978)$ & $0.911(0.834)$ & $0.986(0.972)$ & $0.988(0.976)$ & $0.997(0.994)$ & $\ldots$ \\
q1.5a0 & $0.997(0.994)$ & $0.955(0.906)$ & $0.998(0.995)$ & $0.998(0.995)$ & $0.999(0.997)$ & $\ldots$ \\
q2a0 & $0.999(0.997)$ & $0.973(0.931)$ & $0.994(0.983)$ & $0.994(0.983)$ & $0.997(0.994)$ & $0.999(0.997)$ \\
q3a0 & $0.994(0.990)$ & $0.984(0.971)$ & $0.929(0.841)$ & $0.930(0.842)$ & $0.983(0.963)$ & $0.994(0.994)$ \\
q6a0 & $0.999(0.998)$ & $0.999(0.999)$ & $0.893(0.842)$ & $0.893(0.842)$ & $0.983(0.966)$ & $\ldots$ \\
q1a2 & $0.894(0.844)$ & $0.809(0.701)$ & $0.885(0.822)$ & $0.888(0.826)$ & $0.900(0.850)$ & $\ldots$ \\
q2a2 & $0.986(0.974)$ & $0.947(0.900)$ & $0.992(0.985)$ & $0.994(0.988)$ & $0.985(0.969)$ & $\ldots$ \\
\hline \hline
\end{tabular}



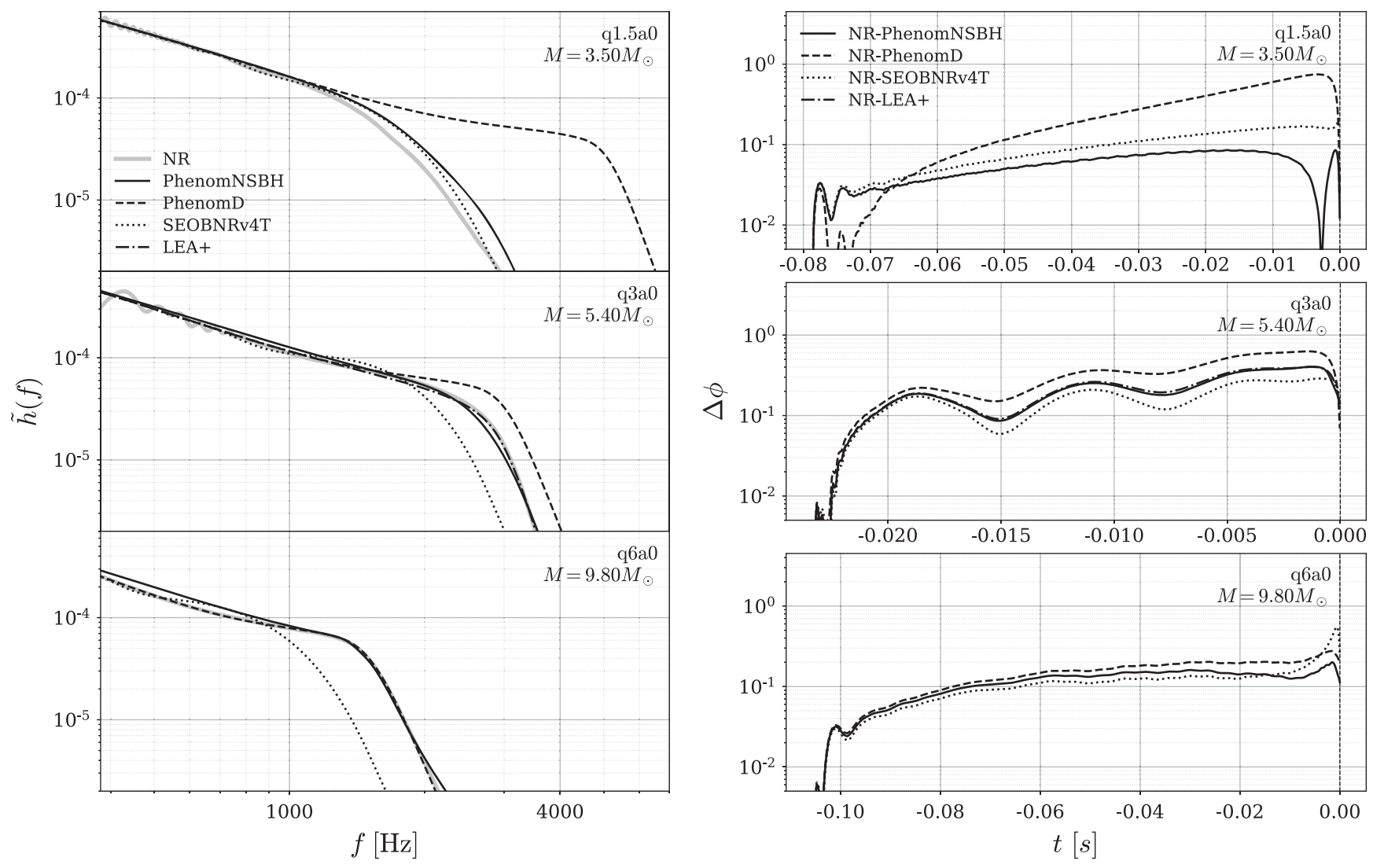

FIG. 1. Comparisons between NR waveforms and various models. The leftmost plots show $\tilde{h}(f)$ for each NR case over the last few orbits before merger, along with frequency-domain representations of the signal using various approximants. The rightmost plots display the accumulated time-domain phase error between the NR phase and each approximant over the length of the NR data, and the approximant signals are aligned by time- and phase-shifts to the NR data over a few GW cycles near the start of each NR simulation. Only the $\mathrm{q} 3 \mathrm{a} 0$ case falls within the parameter space coverage of the LEA+ model.

the accuracy of the model during the late-inspiral and merger.

The results from comparing directly with the NR waveforms are given in Table III, and the faithfulness is computed over the frequency range covered by each NR waveform. We provide results from using the AZDHP (design) noise curve, as well as a flat noise curve (in parentheses). We also compute the faithfulness of several other waveform models to gauge the systematic uncertainty that is incurred by using them. Specifically, we also compare against the NSBH model LEA+ [37], an inspiral NSBH model SEOBNRv4T [20,21], a BBH model PhenomD $[42,43]$ and two inspiral BNS models PhenomDNRT $[24,25,42,43]$ and SEOBNRv4NRT $[24,25,58] .{ }^{1}$

In Ref. [29] the authors analyze the same NR waveforms and the same models. We find similar results and plot these in Fig. 1. Although that work focuses on the agreement

\footnotetext{
${ }^{1}$ The approximant names in the LALSuite code for LEA+, PhenomD, PhenomDNRT, SEOBNRv4T and SEOBNRv4NRT are Lackey_Tidal_2013_SEOBNRV2_ROM, IMRPhenomD, IMRPhenomD_NRTidalv2, SEOBNRv4T and SEOBNRv4_ROM_NRTidā̄ 2 , respectively.
}

between the model and NR by studying the dephasing, here we focus on computing the faithfulness, which is directly related to the loss in signal-to-noise ratio in matched-filter based searches, and takes into account both phase and amplitude differences.

Of the seven NR simulations considered in this paper, five are binary systems without any spin on either body (see Table II for a list of the nonspinning waveforms and their parameters). The two cases including spin, q1a2 and q2a2, are simulations where the NS is spinning with a dimensionless spin magnitude of 0.2 in a direction antiparallel to the orbital angular momentum. The amplitude model used for PhenomNSBH is not calibrated for spinning NSs; however it is constructed to propagate the NS spin through to the uderlying BNS tidal phase model [25] and the underlying BBH amplitude model [41] where the NS spin is treated as a BH spin. These two NR waveforms with spinning NS allow for an exploration of the viability of the model when the NS is spinning. We do not make direct comparisons to NR where the $\mathrm{BH}$ is spinning as no such simulations are currently publicly available. The amplitude model used in this work was calibrated against NSBH NR waveforms with a spinning BH. Furthermore, based on the faithfulness 
TABLE IV. The computed faithfulness between the seven SXS NSBH numerical relativity hybrid waveforms. These have been hybridized with the TEOBResums model with a start frequency of $20 \mathrm{~Hz}$. We compare against the waveform approximants PhenomNSBH, PhenomD, PhenomDnRT, SEOBNRv4T, SEOBNRv4NRT, and LEA+. We compute two sets of matches. The first uses the Advanced LIGO zerodetuning, high-power noise curve and second, in parentheses, uses a flat noise curve. The frequency range used to compute the matches cover the entire bandwidth of the hybrid waveforms, down to a lower frequency bound of $20 \mathrm{~Hz}$.

\begin{tabular}{lcccccc}
\hline \hline Sim name & PhenomNSBH & PhenomD & PhenomDNRT & SEOBNRV4NRT & SEOBNRV4T & LEA+ \\
\hline q1a0 & $0.9996(0.9996)$ & $0.9906(0.9936)$ & $0.9985(0.9989)$ & $0.9992(0.9994)$ & $0.9968(0.9982)$ & $\ldots$ \\
q1.5a0 & $0.9994(0.9997)$ & $0.9930(0.9952)$ & $0.9991(0.9993)$ & $0.9979(0.9984)$ & $0.9973(0.9981)$ & $\ldots$ \\
q2a0 & $0.9987(0.9990)$ & $0.9954(0.9966)$ & $0.9989(0.9993)$ & $0.9969(0.9978)$ & $0.9970(0.9976)$ & $0.9997(0.9998)$ \\
q3a0 & $0.9995(0.9997)$ & $0.9956(0.9975)$ & $0.9990(0.9993)$ & $0.9975(0.9984)$ & $0.9993(0.9995)$ & $0.9990(0.9990)$ \\
q6a0 & $0.9974(0.9981)$ & $0.9964(0.9972)$ & $0.9946(0.9974)$ & $0.9957(0.9972)$ & $0.9977(0.9988)$ & $\ldots$ \\
q1a2 & $0.9969(0.9978)$ & $0.9405(0.9508)$ & $0.9949(0.9967)$ & $0.9962(0.9972)$ & $0.9965(0.9975)$ & $\ldots$ \\
q2a2 & $0.9991(0.9992)$ & $0.9806(0.9837)$ & $0.9985(0.9992)$ & $0.9988(0.9990)$ & $0.9982(0.9989)$ & $\ldots$ \\
\hline \hline
\end{tabular}

comparisons with LEA+, which is also calibrated to and validated against the same NSBH NR waveforms with a spinning $\mathrm{BH}$, we expect the model to also perform well where the $\mathrm{BH}$ is spinning and provide an accurate model for these systems up to numerical errors present in the original calibration set for these models.

For q1a2, when we compare against the BBH model PhenomD the match is 0.809 (0.701) for the AZDHP (flat) noise curve. Including tidal effects in the model does improve the match where we find a match of $\sim 0.89$ ( $\sim 0.84)$ for the AZDHP (flat) noise curve. For q2a2, the match is not as bad as q1a2 but the results are, in general, worse than the nonspinning cases. Comparisons against LEA+ are not included for these two NSBH NR waveforms with a spinning NS as LEA+ does not depend on NS spin.

Reference [29] showed that the NR phase error is smaller than the systematic modelling error in the original NRTidal phase approximant model. Similarly, we also find a noticeable phase difference between the phase description employed in PhenomNSBH and the NR data. These results suggest that further improvements such as a new phase calibration to NSBH NR simulations or the inclusion of spin-dependent f-mode resonance shifts near merger [20] may be important to include. In the next section, however, we show that the measured dephasing is not an issue for Advanced LIGO at design sensitivity.

\section{B. Comparison to hybrid numerical-relativity waveforms}

We now repeat the comparisons performed above, but we use hybridized NR waveforms to test the accuracy of the models for realistic signals including the thousands of inspiral cycles prior to merger. To do this, we produce hybrid waveforms, attaching the SXS NSBH waveforms listed in Table II to the tidal inspiral approximant TEOBResums [19], following the hybridization procedure outlined in $[24,59]$. These hybrids have a starting frequency below $20 \mathrm{~Hz}$ and allow us to test the models in a realistic observational scenario where a current-generation ground- based detector would also be sensitive to the full inspiral from $20 \mathrm{~Hz}$; for the faithfulness integrals we use a low frequency cutoff of $20 \mathrm{~Hz}$. We have verified the accuracy of our hybrid construction method and find that the mismatch of a given hybrid with respect to itself subject to varying the hybridization parameters is $\mathcal{O}\left(10^{-4}\right)$.

We list the results of the faithfulness calculations in Table IV. In general we find that the matches are very high, even when comparing the NSBH hybrids against $\mathrm{BBH}$ models, with the exception of the spinning NSBH waveform q1a2. At the total masses considered here, the signal-to-noise ratio (SNR) detectable in Advanced LIGO is dominated by the long inspiral, and as a result inaccuracies in the waveform model during merger contribute much less to the total SNR. Note also that, as the hybrids were constructed with the TEOBResums model as the inspiral approximant, it is encouraging that we find strong agreement between models with different tidal inspiral approximants.

\section{Importance of NSBH-specific contributions}

The distinguishing difference in the model of an NSBH waveform from a BBH waveform is its behavior close to merger, where strong tidal effects lead to dephasing of the binary from the standard BBH phase and may lead to disruption of the NS, thereby greatly tapering the amplitude. As the total mass of the NSBH system for this model is expected to be relatively low (not exceeding $\sim 45 M_{\odot}$ ), these effects will occur at high frequencies where current ground-based detectors are not highly sensitive. One must then ask how important these effects are to the overall model of the waveform for current and future detectors, and how distinguishable the NSBH-specific effects are from $\mathrm{BBH}$ or BNS systems.

To estimate the importance of tidal effects and disruption for the detectability of an NSBH signal, we compute the SNR at which the NSBH waveform deviates from other waveform approximants covering the parameter space for these merger types; in particular, we compare against 
both PhenomNSBH with $\Lambda=0$ to simulate a purely $\mathrm{BBH}$ waveform and PhenomDNRT, which contains the same phase model as PhenomNSBH but has a taper applied to the high-frequency merger content of the waveform.

Given an NSBH signal with 4 internal degrees-offreedom $(M, q, \chi, \Lambda)$, the SNR $\rho$ associated with a 90\% confidence region in parameter space for detection is related to the faithfulness $\mathcal{F}$ between the NSBH signal (here produced by PhenomNSBH) and another waveform approximant via [60]

$$
\mathcal{F}=1-\frac{3.89}{\rho^{2}} .
$$

We initially compute a series of NSBH waveforms using fixed intrinsic parameters $\left(M_{\mathrm{NS}}, \chi, \Lambda\right)=\left(1.35 M_{\odot}, 0,400\right)$ and allow the mass ratio to vary between 1 and 8 . This ensures that we evaluate all merger types captured by the amplitude model in the comparison.

The SNR resulting from these comparisons is plotted in Fig. 2. Focusing first on the distinguishability SNR between PhenomNSBH and PhenomDNRT, we see that the two models will be easier to distinguish with a modestly loud signal in an Advanced LIGO-type detector as the mass ratio of the system increases. In the NSBH system, the mass scale is fixed by the NS mass and therefore as $q$ increases, so too does the total mass $M$. This increase in $M$ will push the merger regime of the system into a lower (and more sensitive) frequency band in the detector, making the high-frequency taper applied to the NRTidal model more

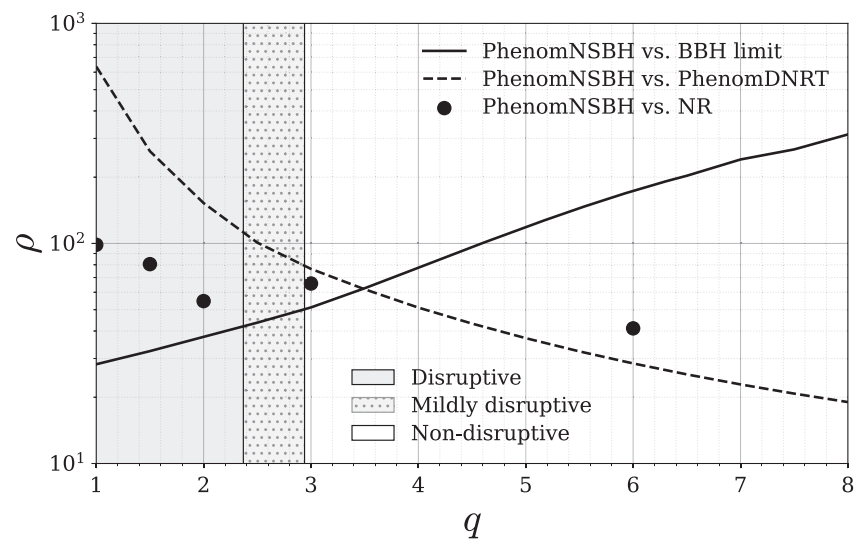

FIG. 2. The approximate SNR at which the waveforms PhenomDNRT and the BBH-limit of PhenomNSBH become distinguishable from PhenomNSBH is plotted as a function of mass-ratio for a nonspinning NSBH system with tidal deformability $\Lambda=400$ and NS mass $1.35 M_{\odot}$. The shaded regions of the plot indicate different merger types calculated from PhenomNSBH. The solid dots show the SNR computed from mismatches between PhenomNSBH and the NR-hybrid data listed in Table IV. The trends continue to higher mass ratios, where an NSBH signal becomes effectively indistinguishable from a $\mathrm{BBH}$ signal in any realistic detection. The matches between models are computed over the range $\left[f_{1}, f_{2}\right]=[25,8192] \mathrm{Hz}$ assuming a AZDHP noise curve. apparent in the faithfulness calculation. At lower $q$ in the disruptive regime of the NSBH system, the taper applied to the NRTidal model mimics the disruption at high frequency in the NSBH waveform. Furthermore, these differences between the two models occur at such high frequency that the lack of sensitivity in the detector makes them hard to distinguish.

We stress that this comparison extends the use of PhenomDNRT well beyond the valid parameter space for a BNS system. We wish to test the usefulness of using the PhenomDNRT model to describe an NSBH system, as these systems can share similar amplitude morphologies depending on NSBH merger type. The relatively low distinguishable SNR seen as the mass-ratio increases is not only caused by the change in NSBH morphology but also due to extension of PhenomDNRT beyond its reliable calibration region.

When looking at the comparison between PhenomNSBH with and without tidal effects (i.e., comparing against a $\mathrm{BBH}$ waveform), we observe the inverse behavior with changing $q$. Even though the disruptive mergers of comparable-mass NSBH binaries lie outside the most sensitive frequency ranges of ground-based detectors, the differences in the waveforms due to tidal effects in the inspiral still allow us to distinguish between $\mathrm{BBH}$ and NSBH systems above SNR of 28. This observation is consistent with GW170817 [9] that had an SNR of 32.4 and allowed us to bound the mass-weighted tidal deformability $\tilde{\Lambda}$ away from zero. As the mass ratio increases, tidal effects scale away as $q^{-4}$ in the phase and the NSBH signal becomes hard to differentiate from a $\mathrm{BBH}$ signal in the nondisruptive regime; the only differences between the two models are the properties of the remnant quantities after merger.

We expand this comparison to include the broader parameter space covered by PhenomNSBH. Specifically, we assume a AZDHP noise curve and calculate the distinguishability SNR between PhenomNSBH and its BBH limit, and between PhenomNSBH and PhenomDNRT for $\sim 5 \times 10^{3} \mathrm{NSBH}$ systems with randomly chosen properties. $R_{\mathrm{NS}}$ is uniformly sampled between $9 \mathrm{~km}$ and $16 \mathrm{~km}$. $M_{\mathrm{NS}}$ is then uniformly sampled over an interval consistent with $\Lambda \in[0,5000]$ that is dependant on $R_{\mathrm{NS}}$ and bounded by $1.0 M_{\odot}$ and $2.3 M_{\odot}$. $\Lambda$ is then calculated from $R_{\mathrm{NS}}, M_{\mathrm{NS}}$ and inverting the universal relation Eq. (4). $q$ is uniformly sampled in the interval $[1,15]$ to give $M_{\mathrm{BH}}$, while the $\mathrm{BH}$ (aligned) spin is uniformly sampled in the interval $[-0.5,0.5]$. Our results are collected in Fig. 3. The top (middle) panel shows the distinguishability SNR values yielded by PhenomNSBH and its BBH limit (PhenomDNRT), while the bottom panel displays the maximum distinguishable SNR between PhenomNSBH and the two other models. We see that the general trend described by Fig. 2 holds. In particular the characteristic SNR minimum at which the most distinguishable waveform model transitions between PhenomDNRT and 


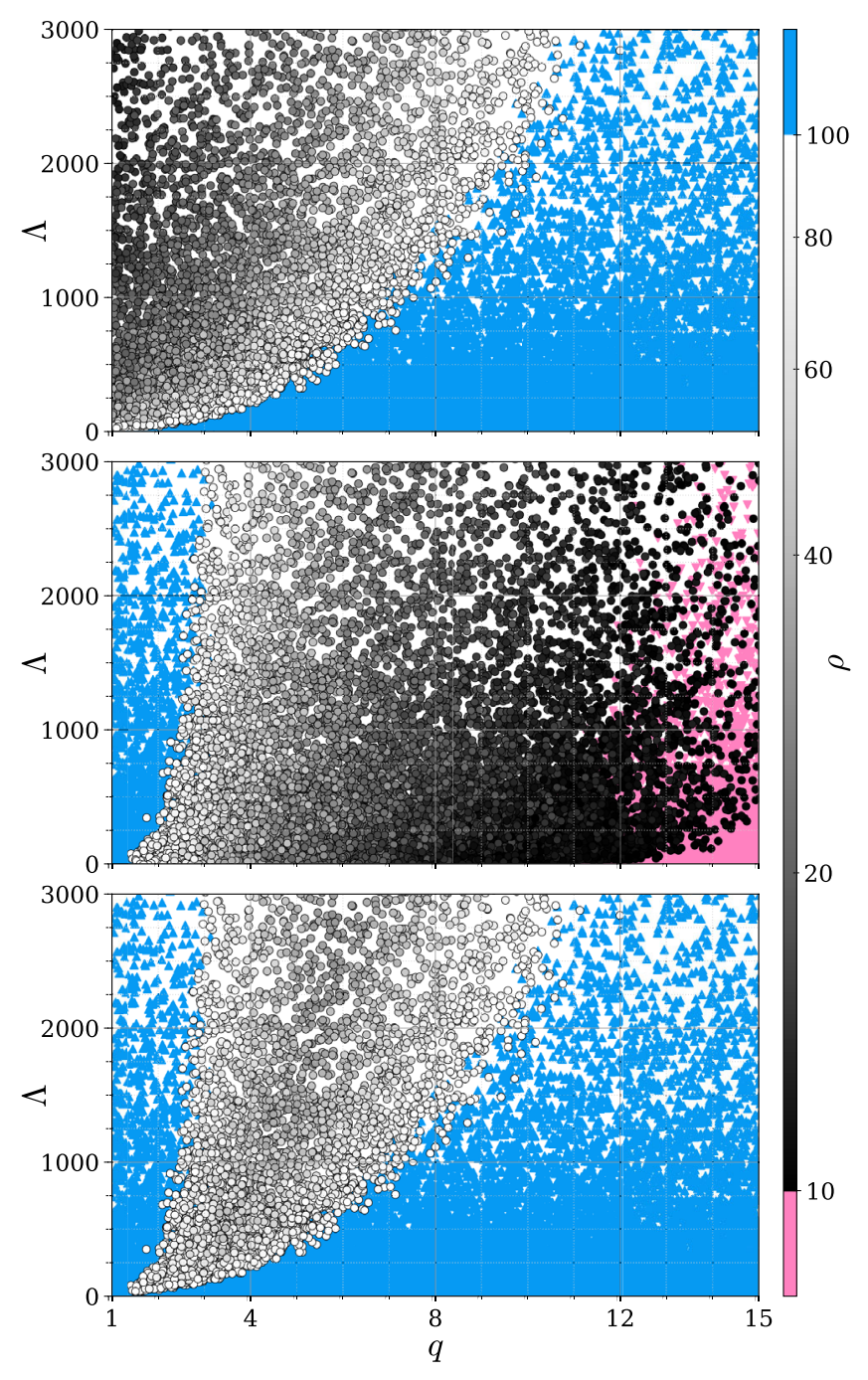

FIG. 3. The approximate SNR at which the waveforms for BNS (PhenomDNRT) and BBH (the BBH-limit of PhenomNSBH) become distinguishable from NSBH (PhenomNSBH), considered over the entire parameter space of PhenomNSBH and projected onto the $q$ - $\Lambda$ plane. The top panel displays the distinguishablity of PhenomNSBH from its $\mathrm{BBH}$-limit, the middle panel the distinguishablity of PhenomNSBH from PhenomDNRT, and the bottom panel the maximum distinguishable SNR between PhenomNSBH and the two other models. Distinguishable SNRs below 10 are displayed as pink upside-down triangles and as blue triangles for SNRs above 100. The AZDHP noise curve is used to compute these results.

the $\mathrm{BBH}$ limit persists across parameter space, widening and deepening as tidal deformability increases.

Similar to the model comparisons with PhenomNSBH already presented, the region where PhenomNSBH can be easily distinguished from PhenomDNRT occurs outside of PhenomDNRT's original parameter bounds and largely results from the extrapolation of the high frequency taper used in the valid region of the BNS model. In contrast PhenomNSBH can be easily distinguished from the BBH limit where both models are valid, and this represents the differences in physical effects modeled by each approximant.
When we consider this transition over the entire parameter space for the model, we find a minimum distinguishable SNR of 27. When constraining $1.35 M_{\odot}<$ $M_{\mathrm{NS}}<1.4 M_{\odot}$ we find the minimum distinguishable SNR only increases slightly to 29 . Constraining $\Lambda<1000$ produces a larger increase in the minimum distinguishable SNR to 35 . Applying both cuts in NS mass and $\Lambda$ increases the minimum distinguishable SNR to 42 . These results indicate that the best chance of distinguishing an NSBH signal with current models is from a system with a particularly stiff EOS. It is in this region of relatively low distinguishable SNR that we expect the NSBH model could be most useful. Assuming a single-detector SNR detection threshold of 10, a minimum distinguishable SNR of $\sim 30$ for an optimally oriented binary system with fixed intrinsic parameters corresponds to a decrease in the distinguishable volume by a factor of $\sim 27$ compared to the detectable volume, and thus roughly one in every 27 NSBH detections of this type could be distinguished from either a BBH or BNS signal.

If a signal were to be detected with SNR $>60$, comparisons with available NR waveforms suggest that systematic errors in the modeling would enter the waveform and would potentially bias any results inferred from using these models. While we do not anticipate signals with such a high SNR to be seen until third-generation detectors [61-63] begin operation, should such a signal be detected we will require more accurate NSBH models and potentially more accurate NR simulations of NSBH systems [29]. However, we have shown that for typical observations we expect either BNS or BBH waveform models to be sufficient.

\section{DISCUSSION}

In this paper we have outlined the construction of PhenomNSBH, an updated waveform model specific to signals from NSBH systems. This model uses an improved amplitude model that identifies distinct merger morphologies and a new tidal phase model, both of which have been calibrated using NR data. The model is valid for systems with mass-ratios ranging from $q \in[1,15]$ with NS masses between $M_{\mathrm{NS}} \in[1,3] M_{\odot}$, BH spins aligned with the orbital angular momentum ranging between $\chi \leq|0.5|$, and NS tidal deformabilities between $\Lambda \in[0,5000]$, though we direct the reader toward discussions about untested regions for this model, which can be found at the beginning of Sec. III. In addition, the model described here performs well when compared against available NSBH NR waveforms with spinning neutron stars, despite the amplitude model lacking such systems in its calibration.

We have shown in Figs. 2 and 3 that the NSBH-specific characteristics of PhenomNSBH are distinguishable from other waveform models in different regions of parameter space. As the merger transitions to the nondisruptive regime, the amplitude of the waveform deviates further from a $\mathrm{BBH}$ waveform amplitude, which will be 
distinguishable in ground-based detectors for moderately loud signals. As the merger type becomes less disruptive, the NSBH waveform will easily be distinguishable from a BNS waveform model (e.g., PhenomDNRT) due to the taper at high frequency applied to the latter and lack of ringdown in the signal. The important conclusion to draw from these results is that for current ground-based detectors, there is only a small region of parameter space where it may be possible to unambiguously identify an NSBH system given current waveform models. This statement is limited to single observations, and to aligned-spin models that include only the dominant waveform harmonic.

The waveform model PhenomNSBH described in this paper is an improvement/extension of current NSBH waveform models, but there is certainly room for future advances. While recent cosmological simulations predict that the majority of NSBH systems will have relatively low mass-ratios $(q \sim[3,5])$ [64], even at these low massratios the effects of higher modes [44,45] and precession [65-67] are important to capture the essential physics from the waveform and should be a primary focus of future NSBH waveform modeling efforts. Another avenue for improvement lies in calibrating the phase model against NSBH NR waveforms. These tasks will require a large catalog of new NR simulations at high resolution and spanning a large range of mass-ratios, spins, and tidal deformability.

\section{ACKNOWLEDGMENTS}

The authors would like to express thanks to Frank Ohme, Andrew Matas, and Shrobana Gosh for their work in reviewing the LALSuite implementation of PhenomNSBH, and John Veitch for discussions that initiated this project. The authors would also like to thank the journal referee for insightful and clarifying comments. J. T. would like to thank Sarp Akcay for assisting with the production of TEOBResums waveforms used in the hybrid generation. T. D. acknowledges support by the European Union's Horizon 2020 research and innovation program under Grant Agreement No. 749145, BNSmergers. J. T. and M. H. were supported by Science and Technology Facilities Council (STFC) Grant No. ST/L000962/1 and thank the Amaldi Research Center for hospitality. J. T., M. H., S. K., and E.F-J were supported by European Research Council Consolidator Grant No. 647839. S. K. acknowledges support by the Max Planck Society's Independent Research Group Grant. Analysis and plots in this paper were made using the PYTHON software packages LALSuite [39], MATPLOTLIB [68], NUMPY [69], РуCBC [70], and SCIPY [71]. The authors are grateful for computational resources provided by the LIGO Laboratory, supported by National Science Foundation Grants No. PHY-0757058 and No. PHY-0823459, and by Cardiff University supported by STFC Grant No. ST/I006285/1.

\section{APPENDIX A: AMPLITUDE MODEL WORKFLOW}

For the convenience of the reader, we now outline the construction of the amplitude model in more detail following the flowchart in Sec. II A. To begin, the compactness of the NS is determined from the input tidal deformability, as described in detail in Appendix B.

We compute the tidal disruption frequency, $f_{\text {tide }}$, which approximates the frequency at which the external quadrupolar tidal force acting on the NS from the companion $\mathrm{BH}$ is comparable in magnitude to the self-gravitating force maintaining the NS. This follows from the initial parameters of the binary according to $[72,73]$

$$
\begin{gathered}
f_{\text {tide }}=\frac{1}{\pi\left(\chi M_{\mathrm{BH}}+\sqrt{\tilde{r}_{\text {tide }}^{3} / M_{\mathrm{BH}}}\right)}, \\
\tilde{r}_{\text {tide }}=\xi_{\text {tide }} M_{\mathrm{BH}} \frac{(1-2 C)}{\mu},
\end{gathered}
$$

where $\mu=q C$ and $\xi_{\text {tide }}$ is the largest positive real root of the following equation:

$$
\begin{aligned}
0= & \xi_{\text {tide }}^{5}-3 \mu \xi_{\text {tide }}^{4}+2 \chi \sqrt{\mu^{3} \xi_{\text {tide }}^{7}}-3 q \xi_{\text {tide }}^{2} \\
& +6 q \mu \xi_{\text {tide }}-3 q \mu^{2} \chi^{2}
\end{aligned}
$$

Next, the ratio of the baryonic mass of the torus remaining after merger to the initial baryonic mass of the NS, $M_{b, \text { torus }} / M_{b, \mathrm{NS}}$, is determined according to fits from [72],

$$
\frac{M_{b, \text { torus }}}{M_{b, \mathrm{NS}}}=0.296 \xi_{\text {tide }}(1-2 C)-0.171 q C \bar{r}_{\mathrm{ISCO}},
$$

where $\bar{r}_{\text {ISCO }}$ is the radius of the innermost stable circular orbit of a unit-mass BH [74],

$$
\begin{aligned}
\bar{r}_{\mathrm{ISCO}} & =\left[3+Z_{2}-\operatorname{sign}(\chi) \sqrt{\left(3-Z_{1}\right)\left(3+Z_{1}+2 Z_{2}\right)}\right] \\
Z_{1} & =1+\left(1-\chi^{2}\right)^{1 / 3}\left[(1+\chi)^{1 / 3}+(1-\chi)^{1 / 3}\right], \\
Z_{2} & =\sqrt{3 \chi^{2}+Z_{1}^{2}} .
\end{aligned}
$$

The fit for $M_{b, \text { torus }}$ was recently updated in Ref. [75]; incorporating it in the amplitude model would require recalibrating the NSBH amplitude model itself as a whole and we leave this for future work.

The final mass, $M_{f}$, and final spin, $\chi_{f}$, of the remnant $\mathrm{BH}$ after merger are calculated using NSBH-specific fits for the remnant properties parametrized by tidal deformability [76], 


$$
\begin{gathered}
F(\eta, \chi, \Lambda)=F_{\mathrm{BBH}}(\eta, \chi) \frac{1+p_{1}(\eta, \chi) \Lambda+p_{2}(\eta, \chi) \Lambda^{2}}{\left(1+\left[p_{3}(\eta, \chi)\right]^{2} \Lambda\right)^{2}} \\
p_{k}(\eta, \chi)=p_{k 1}(\chi) \eta+p_{k 2}(\chi) \eta^{2} \\
p_{k j}(\eta, \chi)=p_{k j 0} \chi+p_{k j 1} .
\end{gathered}
$$

The remnant model $F_{\mathrm{BBH}}$ is the model for the final mass and spin of a BBH coalescence described in [77], and the coefficients $p_{k j i}$ for the final mass $M_{f}$ and final spin $\chi_{f}$ can be found in the Supplemental Material for [76]. Once the final mass and spin are determined, we find the ringdown frequency $f_{\mathrm{RD}}$ and quality factor $Q$ via

$$
\begin{gathered}
f_{\mathrm{RD}}=\frac{\Re(\tilde{\omega})}{2 \pi M_{f}}, \\
Q=\frac{\Re(\tilde{\omega})}{2 \Im(\tilde{\omega})},
\end{gathered}
$$

where $\tilde{\omega}$ is a fit to the $(l, m, n)=(2,2,0)$ Kerr quasinormal mode frequency given in [78],

$$
\begin{gathered}
\tilde{\omega}(\kappa)=1.0+1.5578 e^{2.9031 i} \kappa \\
+1.9510 e^{5.9210 i} \kappa^{2}+2.0997 e^{2.7606 i} \kappa^{3} \\
+1.4109 e^{5.9143 i} \kappa^{4}+0.4106 e^{2.7952 i} \kappa^{5}, \\
\kappa\left(\chi_{f}\right)=\sqrt{\log _{3}\left(2-\chi_{f}\right)} .
\end{gathered}
$$

The amplitude ansatz in Eq. (4) uses the merger-typedependent frequencies $\tilde{f}_{0}, \tilde{f}_{1}$, and $\tilde{f}_{2}$ to blend the postNewtonian, premerger, and merger-ringdown amplitude contributions together. These frequencies are determined based on the conditions in Table I. We now list the specific functional form of the various component functions $x_{\mathrm{ND}}$, $x_{\mathrm{ND}}^{\prime}, x_{\mathrm{D}}$ and $x_{\mathrm{D}}^{\prime}$ of the merger-type dependent quantities given in [38]. The nondisruptive fitting functions $x_{\mathrm{ND}}$ and $x_{\mathrm{ND}}^{\prime}$ also require the scaled ringdown frequency $\tilde{f}_{\mathrm{RD}}$ calculated according to

$$
\begin{aligned}
& \tilde{f}_{\mathrm{RD}}= \begin{cases}0.99 \times 0.98 f_{\mathrm{RD}}, & \Lambda>1 \\
\left(1-0.02 \Lambda+0.01 \Lambda^{2}\right) \times 0.98 f_{\mathrm{RD}}, & \Lambda \leq 1,\end{cases} \\
& x_{\mathrm{ND}}=\left(\frac{f_{\mathrm{tide}}-\tilde{f}_{\mathrm{RD}}}{\tilde{f}_{\mathrm{RD}}}\right)^{2}-0.571505 C-0.00508451 \chi,
\end{aligned}
$$

$$
x_{\mathrm{D}}=\frac{M_{b, \mathrm{torus}}}{M_{b, \mathrm{NS}}}+0.424912 C+0.363604 \sqrt{\eta}-0.060559 \chi,
$$

$$
\begin{aligned}
x_{\mathrm{D}}^{\prime}= & \frac{M_{b, \text { torus }}}{M_{b, \mathrm{NS}}}-0.132754 C+0.576669 \sqrt{\eta} \\
& -0.0603749 \chi-0.0601185 \chi^{2} \\
& -0.0729134 \chi^{3}
\end{aligned}
$$

The amplitude component function for the inspiral, $A_{\mathrm{PN}}$, is given by the Fourier transform of the time-domain amplitude given in Eq. (3.14) of [41] using the stationary phase approximation,

$$
A_{\mathrm{PN}}(x)=\sqrt{\frac{2 \pi}{3 \dot{x} \sqrt{x}}} 8 \eta x \sqrt{\frac{\pi}{5}} \sum_{k=0}^{6} \mathcal{A}_{k} x^{k / 2},
$$

where $x=\omega^{2 / 3}, \omega$ is the orbital angular frequency of the binary, and $\dot{x}$ is computed using the TaylorT4 expansion [79]; see [41] for the expansion coefficients $\mathcal{A}_{i}$.

The phenomenological correction parameter $\gamma_{1}^{\prime}$ for the premerger region is calculated according to

$$
\gamma_{1}^{\prime}= \begin{cases}1.25, & \Lambda>1 \\ 1-0.5 \Lambda-0.25 \Lambda^{2}, & \Lambda \leq 1,\end{cases}
$$

where the piecewise definition split at $\Lambda=1$ is used to smoothly match to the BBH limit where $\Lambda=0$.

The merger-ringdown component function $A_{\mathrm{RD}}$ is defined by [38]

$$
\begin{gathered}
A_{\mathrm{RD}}(f)=\epsilon_{\text {tide }} \delta_{1} \frac{\sigma^{2}}{\left(f-f_{\mathrm{RD}}\right)^{2}+\sigma^{2} / 4} f^{-7 / 6}, \\
\sigma=\delta_{2}^{\prime} f_{\mathrm{RD}} / Q
\end{gathered}
$$

where the phenomenological correction parameter $\delta_{2}^{\prime}$ is calculated according to a piecewise definition to smoothly match to the $\mathrm{BBH}$ limit as is done for $\gamma_{1}^{\prime}$,

$$
\delta_{2}^{\prime}= \begin{cases}\frac{A}{2} \omega_{x_{3}, d_{3}}^{-}\left(\frac{f_{\text {tide }}-\tilde{f}_{\mathrm{RD}}}{\tilde{f}_{\mathrm{RD}}}\right), & \Lambda>1 \\ \delta_{2}-2\left(\delta_{2}-b_{0}\right) \Lambda+\left(\delta_{2}-b_{0}\right) \Lambda^{2}, & \Lambda \leq 1\end{cases}
$$

with $A=1.62496, x_{3}=0.0188092$, and $d_{3}=0.338737$, $b_{0}=0.81248$ and $\omega_{f_{0}, d}^{ \pm}(f)$ is a hyperbolic tangent windowing function,

$$
\omega_{f_{0}, d}^{ \pm}(f)=\frac{1}{2}\left[1 \pm \tanh \left(\frac{4\left(f-f_{0}\right)}{d}\right)\right] .
$$

Note that the factor of $1 / 2$ multiplying the windowing function $\omega_{x_{3}, d_{3}}^{-}$in Eq. (A22) corrects a typographical error 
in [38]. The PhenomC phenomenological parameters $\delta_{1}, \delta_{2}$ and $\gamma_{1}$ are given as an expansion in symmetric mass-ratio and spins by

$$
\delta_{1}, \delta_{2}, \gamma_{1} \sim \sum_{i+j \in\{1,2\}} \zeta^{i j} \eta^{i} \chi^{j}
$$

with the coefficients $\zeta^{i j}$ in the $\delta_{1}, \delta_{2}$, and $\gamma_{1}$ fit parameters given in [41]. We impose the addition constraints that $\delta_{1}$, $\gamma_{1} \geq 0$ and $\delta_{2} \geq 10^{-4}$ to ensure that the amplitude function Eq. (4) remains positive for all regions of parameter space that PhenomNSBH is expected to be used in. It is necessary to invoke these constraints on these coefficients in the nonspinning limit for $q>25$ and $q>15$ for spinning cases. In this region the model no long remains sensible, and comparisons between other BBH waveforms break down. This constraint on the coefficients motivates the suggested upper bound placed on the mass ratio for the parameter space of the model.

\section{APPENDIX B: REPLACING EQUATION OF STATE}

Removing explicit EOS-dependence from the NSBH amplitude model is achieved by finding the compactness $C$

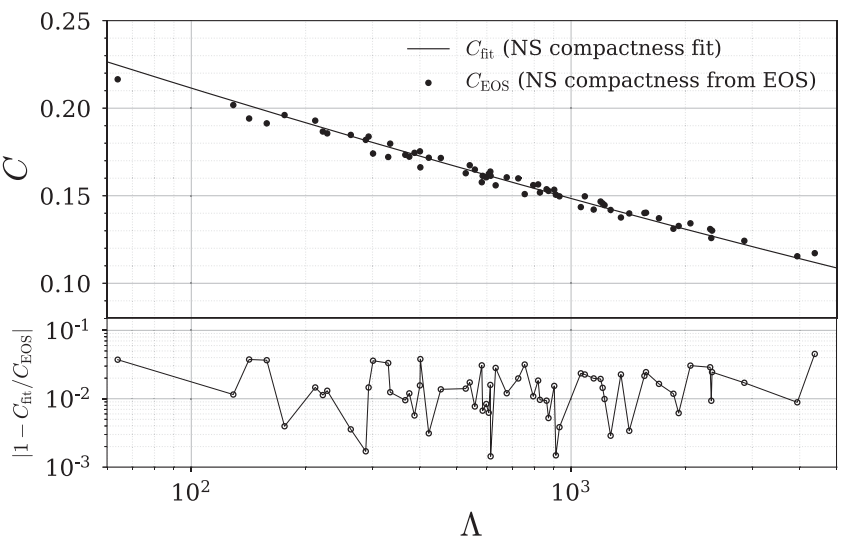

FIG. 4. Comparison between the NS compactness as calculated from the EOS information presented in [37] and the NS compactness fit from [80] which has a root-mean-squared relative percentage error of $1.95 \%$ and maximum relative percentage error of $4.52 \%$.

of the NS from its tidal deformability parameter $\Lambda$ using the fit determined in Ref. [80] with an additional piecewise component for $\Lambda \leq 1$ from [81] to smoothly match to the BBH limit,

$$
C(\Lambda)= \begin{cases}a_{0}+a_{1} \log \Lambda+a_{2}(\log \Lambda)^{2}, & \Lambda>1 \\ 0.5+\left(3 a_{0}-a_{1}-1.5\right) \Lambda^{2}+\left(a_{1}-2 a_{0}+1\right) \Lambda^{3}, & \Lambda \leq 1,\end{cases}
$$

where $a_{0}=0.360, a_{1}=-0.0355$, and $a_{2}=0.000705$. In Fig. 4 we show how the compactness values yielded by this fit compare to those directly obtained from the EOS information presented in [37] by integrating the TolmanOppenheimer-Volkoff equations [48-50].

As the original model was calibrated only to a specific set of EOSs, replacing EOS-dependence with the fit in Eq. (B1) will invariable introduce some error to the amplitude model. We conservatively estimate the effects of this error on the model in the following way.

The error in the fit model is given pessimistically as a $6 \%$ error in the computed value of $C$ across realistic NS EOSs
[80]; for the EOSs used in the calibration of the amplitude model, the error in the fit is bounded by $5 \%$. We invert the mapping in Eq. (B1) and compute the spread in $\Lambda$ produced around a given $\Lambda_{0}$ by varying the compactness within the $6 \%$ error bounds. We then compute matches across the parameter space of PhenomNSBH between two waveforms with all parameters equal except the tidal deformability, which is fixed at $\Lambda_{0}$ for one waveform and allowed to vary between the bounds determined from the compactness error for the other. After sampling waveforms across the model's parameter space, we find a maximum mismatch given by $\sim 10^{-3}$ for the pessimistic $6 \%$ error estimate in the fit.
[1] J. Aasi et al. (LIGO Scientific Collaboration), Classical Quantum Gravity 32, 074001 (2015).

[2] F. Acernese et al. (VIRGO Collaboration), Classical Quantum Gravity 32, 024001 (2015).

[3] M. Vallisneri, J. Kanner, R. Williams, A. Weinstein, and B. Stephens, J. Phys. Conf. Ser. 610, 012021 (2015).
[4] R. Abbott et al. (T. L. S. Collaboration, the Virgo Collaboration), arXiv:1912.11716.

[5] B. P. Abbott et al. (LIGO Scientific, Virgo Collaborations), Phys. Rev. X 9, 031040 (2019).

[6] T. Venumadhav, B. Zackay, J. Roulet, L. Dai, and M. Zaldarriaga, Phys. Rev. D 101, 083030 (2020). 
[7] A. H. Nitz, T. Dent, G. S. Davies, S. Kumar, C. D. Capano, I. Harry, S. Mozzon, L. Nuttall, A. Lundgren, and M. Tápai, Astrophys. J. 891, 123 (2020).

[8] A. H. Nitz, A. B. Nielsen, and C. D. Capano, Astrophys. J. 876, L4 (2019).

[9] B. P. Abbott et al. (LIGO Scientific, Virgo Collaborations), Phys. Rev. X 9, 011001 (2019).

[10] B. P. Abbott et al. (LIGO Scientific, Virgo Collaborations), Astrophys. J. Lett. 892, L3 (2020).

[11] M. W. Coughlin and T. Dietrich, Phys. Rev. D 100, 043011 (2019).

[12] K. Kyutoku, S. Fujibayashi, K. Hayashi, K. Kawaguchi, K. Kiuchi, M. Shibata, and M. Tanaka, Astrophys. J. 890, L4 (2020).

[13] R. Cotesta, A. Buonanno, A. Bohé, A. Taracchini, I. Hinder, and S. Ossokine, Phys. Rev. D 98, 084028 (2018).

[14] S. Khan, K. Chatziioannou, M. Hannam, and F. Ohme, Phys. Rev. D 100, 024059 (2019).

[15] S. Khan, F. Ohme, K. Chatziioannou, and M. Hannam, Phys. Rev. D 101, 024056 (2020).

[16] V. Varma, S. E. Field, M. A. Scheel, J. Blackman, D. Gerosa, L. C. Stein, L. E. Kidder, and H. P. Pfeiffer, Phys. Rev. Research 1, 033015 (2019).

[17] V. Varma, S. E. Field, M. A. Scheel, J. Blackman, L. E. Kidder, and H. P. Pfeiffer, Phys. Rev. D 99, 064045 (2019).

[18] É. É. Flanagan and T. Hinderer, Phys. Rev. D 77, 021502(R) (2008).

[19] A. Nagar et al., Phys. Rev. D 98, 104052 (2018).

[20] T. Hinderer, A. Taracchini, F. Foucart, A. Buonanno, J. Steinhoff, M. Duez, L. E. Kidder, H. P. Pfeiffer, M. A. Scheel, B. Szilagyi et al., Phys. Rev. Lett. 116, 181101 (2016).

[21] J. Steinhoff, T. Hinderer, A. Buonanno, and A. Taracchini, Phys. Rev. D 94, 104028 (2016).

[22] B. D. Lackey, M. Pürrer, A. Taracchini, and S. Marsat, Phys. Rev. D 100, 024002 (2019).

[23] T. Dietrich, S. Bernuzzi, and W. Tichy, Phys. Rev. D 96, 121501(R) (2017).

[24] T. Dietrich et al., Phys. Rev. D 99, 024029 (2019).

[25] T. Dietrich, A. Samajdar, S. Khan, N. K. JohnsonMcDaniel, R. Dudi, and W. Tichy, Phys. Rev. D 100, 044003 (2019).

[26] J. A. Clark, A. Bauswein, N. Stergioulas, and D. Shoemaker, Classical Quantum Gravity 33, 085003 (2016).

[27] K. W. Tsang, T. Dietrich, and C. Van Den Broeck, Phys. Rev. D 100, 044047 (2019).

[28] M. Breschi, S. Bernuzzi, F. Zappa, M. Agathos, A. Perego, D. Radice, and A. Nagar, Phys. Rev. D 100, 104029 (2019).

[29] F. Foucart et al., Phys. Rev. D 99, 044008 (2019).

[30] R. Dudi, F. Pannarale, T. Dietrich, M. Hannam, S. Bernuzzi, F. Ohme, and B. Brügmann, Phys. Rev. D 98, 084061 (2018).

[31] F. Pannarale, L. Rezzolla, F. Ohme, and J. S. Read, Phys. Rev. D 84, 104017 (2011).

[32] T. Yamamoto, M. Shibata, and K. Taniguchi, Phys. Rev. D 78, 064054 (2008).

[33] F. Foucart, L. Buchman, M. D. Duez, M. Grudich, L. E. Kidder, I. MacDonald, A. Mroue, H. P. Pfeiffer, M. A. Scheel, and B. Szilagyi, Phys. Rev. D 88, 064017 (2013).
[34] K. Kyutoku, H. Okawa, M. Shibata, and K. Taniguchi, Phys. Rev. D 84, 064018 (2011).

[35] K. Kyutoku, M. Shibata, and K. Taniguchi, Phys. Rev. D 82, 044049 (2010); 84, 049902(E) (2011).

[36] F. Pannarale, E. Berti, K. Kyutoku, B. D. Lackey, and M. Shibata, Phys. Rev. D 92, 081504 (2015).

[37] B. D. Lackey, K. Kyutoku, M. Shibata, P. R. Brady, and J. L. Friedman, Phys. Rev. D 89, 043009 (2014).

[38] F. Pannarale, E. Berti, K. Kyutoku, B. D. Lackey, and M. Shibata, Phys. Rev. D 92, 084050 (2015).

[39] LIGO Scientific Collaboration, LIGO Algorithm Library LALSuite, free software (GPL) (2018), https://doi.org/ 10.7935/GT1W-FZ16.

[40] T. Hinderer, Astrophys. J. 677, 1216 (2008).

[41] L. Santamaria et al., Phys. Rev. D 82, 064016 (2010).

[42] S. Husa, S. Khan, M. Hannam, M. Pürrer, F. Ohme, X. J. Forteza, and A. Bohé, Phys. Rev. D 93, 044006 (2016).

[43] S. Khan, S. Husa, M. Hannam, F. Ohme, M. Pürrer, X. J. Forteza, and A. Bohé, Phys. Rev. D 93, 044007 (2016).

[44] V. Varma and P. Ajith, Phys. Rev. D 96, 124024 (2017).

[45] C. Kalaghatgi, M. Hannam, and V. Raymond, Phys. Rev. D 101, 103004 (2020).

[46] C. García-Quirós, M. Colleoni, S. Husa, H. Estellés, G. Pratten, A. Ramos-Buades, M. Mateu-Lucena, and R. Jaume, arXiv:2001.10914.

[47] J. S. Read, C. Markakis, M. Shibata, K. b. o. Uryū, J. D. E. Creighton, and J. L. Friedman, Phys. Rev. D 79, 124033 (2009).

[48] R. C. Tolman, Proc. Natl. Acad. Sci. U.S.A. 20, 169 (1934).

[49] R. C. Tolman, Phys. Rev. 55, 364 (1939).

[50] J. R. Oppenheimer and G. M. Volkoff, Phys. Rev. 55, 374 (1939).

[51] B. D. Lackey and L. Wade, Phys. Rev. D 91, 043002 (2015).

[52] F. Pannarale, E. Berti, K. Kyutoku, and M. Shibata, Phys. Rev. D 88, 084011 (2013).

[53] K. Chakravarti et al., Phys. Rev. D 99, 024049 (2019).

[54] https://dcc.ligo.org/LIGO-T0900288/public.

[55] A. Taracchini, Y. Pan, A. Buonanno, E. Barausse, M. Boyle, T. Chu, G. Lovelace, H. P. Pfeiffer, and M. A. Scheel, Phys. Rev. D 86, 024011 (2012).

[56] A. Taracchini, A. Buonanno, Y. Pan, T. Hinderer, M. Boyle, D. A. Hemberger, L. E. Kidder, G. Lovelace, A. H. Mroué, H. P. Pfeiffer et al., Phys. Rev. D 89, 061502 (2014).

[57] F. Ohme, M. Hannam, and S. Husa, Phys. Rev. D 84, 064029 (2011).

[58] A. Bohé, L. Shao, A. Taracchini, A. Buonanno, S. Babak, I. W. Harry, I. Hinder, S. Ossokine, M. Pürrer, V. Raymond et al., Phys. Rev. D 95, 044028 (2017).

[59] K. Hotokezaka, K. Kyutoku, Y.-I. Sekiguchi, and M. Shibata, Phys. Rev. D 93, 064082 (2016).

[60] E. Baird, S. Fairhurst, M. Hannam, and P. Murphy, Phys. Rev. D 87, 024035 (2013).

[61] M. Punturo et al., Classical Quantum Gravity 27, 084007 (2010).

[62] S. Hild et al., Classical Quantum Gravity 28, 094013 (2011).

[63] B. P. Abbott et al. (LIGO Scientific Collaboration), Classical Quantum Gravity 34, 044001 (2017).

[64] M. Mapelli and N. Giacobbo, Mon. Not. R. Astron. Soc. 479, 4391 (2018). 
[65] T. A. Apostolatos, C. Cutler, G. J. Sussman, and K. S. Thorne, Phys. Rev. D 49, 6274 (1994).

[66] T. A. Apostolatos, Phys. Rev. D 52, 605 (1995).

[67] L. E. Kidder, C. M. Will, and A. G. Wiseman, Phys. Rev. D 47, 3281 (1993).

[68] J. D. Hunter, Comput. Sci. Eng. 9, 90 (2007).

[69] S. van der Walt, S. C. Colbert, and G. Varoquaux, Comput. Sci. Eng. 13, 22 (2011).

[70] A. Nitz, I. Harry, D. Brown, C. M. Biwer, J. Willis, T. D. Canton, C. Capano, L. Pekowsky, T. Dent, A. R. Williamson et al., gwastro/pycbc: Pycbc release v1.15.4, https://doi.org/ 10.5281/zenodo.3630601 (2020).

[71] T. E. Oliphant, Comput. Sci. Eng. 9, 10 (2007).

[72] F. Foucart, Phys. Rev. D 86, 124007 (2012).

[73] M. Shibata and K. Taniguchi, Phys. Rev. D 77, 084015 (2008).
[74] J. M. Bardeen, W. H. Press, and S. A. Teukolsky, Astrophys. J. 178, 347 (1972).

[75] F. Foucart, T. Hinderer, and S. Nissanke, Phys. Rev. D 98, 081501 (2018).

[76] F. Zappa, S. Bernuzzi, F. Pannarale, M. Mapelli, and N. Giacobbo, Phys. Rev. Lett. 123, 041102 (2019).

[77] X. Jiménez-Forteza, D. Keitel, S. Husa, M. Hannam, S. Khan, and M. Pürrer, Phys. Rev. D 95, 064024 (2017).

[78] L. London and E. Fauchon-Jones, Classical Quantum Gravity 36, 235015 (2019).

[79] A. Buonanno, Y.-b. Chen, and M. Vallisneri, Phys. Rev. D 67, 104025 (2003); 74, 029904(E) (2006).

[80] K. Yagi and N. Yunes, Phys. Rep. 681, 1 (2017).

[81] A. Matas, A. Buonanno, T. Dietrich, and T. Hinderer, arXiv:2004.10001. 